\title{
The Effect of Leader Communication Strategy on Employee Task and Contextual Performance: The Mediating Role of Feedback Quality
}

\author{
Wen $\mathrm{Xu}^{1, \mathrm{a}}$, Jin $\mathrm{Xue}^{2, \mathrm{~b}}$ \\ ${ }^{1}$ Unit 3, no. 1, zhixin garden, dalian city, liaoning province, China \\ ${ }^{2}$ Unit 2, Shahekou District 5 , dalian city, liaoning province, China \\ a47517105@qq.com belebrity106@sina.com
}

\begin{abstract}
Keywords: leader communication strategy; leader motivating language; feedback quality; task performance; contextual performance
\end{abstract}

\begin{abstract}
Based uponsocial information processing theory and organizational communication theory, the present research examined how leader communication strategy, as measured by leader motivating language, influenced employees' task and contextual performance from a process mechanism of feedback quality. The research employed Mplus software to analyzea sample of 237 supervisorsubordinate dyadic data. The finding indicated that (1)leader motivating language had positive effects on both task and contextual performance; (2) and it also had a positive influence on employee perceived feedback quality; (3) meanwhile feedback quality could significantly mediated the relationship between leader motivating language and contextual performance but not significantly mediated the relationship between leader motivating language and task performance.
\end{abstract}

\section{Introduction}

According to the theory of organizational communication, language and social interaction can significantly influence the realization of organizational goals and effectiveness, which results from the motivational component contained in speech messages. Previous studies believed that LML could help subordinates to clarify the direction of work and reduce uncertainty, build the meaning in the work scene and bring emotional care and encouragement. These communication strategies have become the motivation elements to motivate employees' individual behaviors. LML can positively influence employees' work performance, job satisfaction and organizational commitment, retention intention, self-efficacy and team innovation performance, and reduce employees' absence from work. Although leader-member exchange and self-efficacy can to some extent explain the effect of LML on employee performance, the process characteristics of LML as a leadership communication strategy are theoretically ignored. Therefore, based on the social information processing theory, this study proposes an intermediary mechanism for LML to influence employees' work performance. It is believed that the performance of the motivational efficiency of leadership speech requires the leader to give subordinates high-quality information feedback process, and improve the social information communication channels by constructing a high-quality feedback environment, so as to improve the performance of employees.

\section{Research design}

\subsection{Research hypothesis}

\subsubsection{Motivational speech and employee performance}

In the workplace, leadership speech behavior is a key communication strategy that affects employees' work behavior, because the development of leadership is reflected in the discourse content and communication behavior expressed by leaders to subordinates. LML is a communication strategy focusing on improving and motivating employees' work motivation. Employees' perception and understanding of leaders' speech content will be translated into specific work content, and their positive attitude and commitment to the organization will be improved. Motivational speech is an important communication strategy to motivate employees' work motivation. The motivational 
component behind leadership speech becomes the basis of other leadership behaviors, such as transformational leadership, leader-member interaction and goal path model. Based on the motivational speech theory, this study believes that LML can significantly improve the task performance and peripheral performance of employees. The following hypotheses are proposed in this study.

Hypothesis 1: LML has a positive correlation with employee task performance.

Hypothesis 2: LML has a positive correlation with employee peripheral performance.

\subsubsection{Mediating effect of feedback quality}

This study focused on feedback quality as an intermediary mechanism to explain LML's promotion of employee task performance and peripheral performance, and explored how words of leadership motivation can promote and improve the work performance of subordinates from the theoretical framework of organizational communication. Feedback quality is an important index to reflect the feedback environment of an organization. What LML and feedback have in common is that both can enhance employees' motivation to work. The motivation basis of employee performance comes from motivational speech expressed by leaders and high-quality verbal feedback.

Hypothesis 3: LML has a positive correlation with feedback quality perceived by employees.

The contextual information implied by feedback quality can further improve the task performance and peripheral performance of employees. Previous studies have shown that organizational feedback environment is positively correlated with employee task performance, organizational citizenship behavior and job satisfaction. This study puts forward the research hypothesis of this stage.

Hypothesis 4: the perceived feedback quality of employees can mediate the positive correlation between LML and employee task performance.

Hypothesis 5: the perceived feedback quality of employees can mediate the positive correlation between LML and surrounding performance of employees.

\subsection{Research methods}

\subsubsection{Samples}

In this study, enterprises in the Yangtze river delta region were selected as research samples, and a total of 350 sets of questionnaires were distributed. Finally, 237 sets of valid questionnaires were recovered (the recovery rate was $67.71 \%$ ). Each set of data matched the leaders and subordinates with a sample ratio of 1:2.3. Through demographic analysis of the data, the sample characteristics of employees include: $51.1 \%$ of male employees, 29.65 years of overall average age $(\mathrm{SD}=5.69), 5.78$ years of average working age $(\mathrm{SD}=4.51)$, and $92.8 \%$ of them have received education from high school, college and university. The sample characteristics of leaders included: male leaders accounted for 51.9 percent, the overall average age was 36.03 years $(\mathrm{SD}=7.05)$, the average working age was 11.55 years $(\mathrm{SD}=6.91), 12.8$ percent, 21.4 percent, 27.8 percent, 26.9 percent and 11.1 percent had received junior high school, senior high school, junior college, undergraduate and graduate school respectively. 51.7 percent of the sample came from private companies, with the rest coming from foreign companies.

\subsubsection{Measuring tools}

In this study, enterprises in the Yangtze river delta region were selected as research samples, and a total of 350 sets of questionnaires were distributed. Finally, 237 sets of valid questionnaires were recovered (the recovery rate was $67.71 \%$ ). Each set of data matched the leaders and subordinates with a sample ratio of 1:2.3.

\subsubsection{Measuring tools}

(1) Motivational speech of leadership

Confirmatory factor analysis showed that LML of the second-order structure had a good fitting index, suggesting that the three-dimension structure could well reflect the validity of the speech of leadership motivation. The Cronbach's alpha coefficient of the total questionnaire was 0.89 , and the coefficients of the three sub-questionnaires were 0.84 for direction guidance, 0.78 for transference speech and 0.83 for meaning endowment. 
(2) Feedback quality

Confirmatory factor analysis shows that the feedback quality has an acceptable fitting index, which is as follows: as for the first two indicators, it is relatively low, but it is considered that the overall structural validity of the feedback index can be acceptable in combination with other indicators. The Cronbach's alpha coefficient of the questionnaire was 0.74 .

(3) Task performance

The confirmatory factor analysis shows that the fitting index of task performance is very good, as follows: alias 2(5)=9.52, p>.05, RMSEA=.06, SRMR=.02, CFI=.99, TLI=.98. The Cronbach's alpha coefficient of the questionnaire is 0.86 .

(4) Peripheral performance

The confirmatory factor analysis indicates that the surrounding performance has a good fitting index, which is as follows: gear 2(2)=6.39, p=.04, RMSEA=.09, SRMR=.02, CFI=.99, TLI=.98. The Cronbach's alpha coefficient of the questionnaire is 0.89 .

\subsubsection{Analysis strategy}

In this study, the four-factor structure model was proposed as the hypothesis model (LML of secondorder structure, feedback quality, task performance and peripheral performance), and the matching degree difference between the model and other alternative models was compared to obtain the test evidence of discriminant validity. Then, the mediation model of this study was tested, and the fitting indexes of the complete and partial mediation models were compared and selected. Meanwhile, boostrapping is used to estimate the mediating effect of the model, and the confidence interval of $99 \%$ significance level is given. The model fitting index is mainly based on the chi-squared value and chisquared degree of freedom ratio. < 2), CFI (Comparative Index, the Fit. > 90), TLI (Tucker - Index Lewis, > 90), RMSEA (Root Mean Square Error of Approximation, <. 05 or <. 08) and SRMR (Standardized Root Mean Square Residual, <. 05 or <. 08), etc.

\section{Research results}

\subsection{Distinguish validity from descriptive statistics}

The hypothesis model proposed in this study includes LML, feedback quality, task performance and peripheral performance of second-order structure. Through structural equation model analysis, it is shown that this hypothesis model has a better fitting index than other alternative models, with $2(221)=338.50, \mathrm{p}<.001,2 / \mathrm{df}=1.53, \mathrm{RMSEA}=.05, \mathrm{SRMR}=.05, \mathrm{CFI}=.96, \mathrm{TLI}=95$. Assumptions of the model fitting level significantly higher than that of contains four factors model of the single dimension LML $(\Delta$ chi square $(3)=165.09, \mathrm{p}<.001)$, three factors model $(\Delta$ chi square $(3) \quad \mathrm{I}=427.15, \mathrm{p}<$. 001 ; II $\Delta$ chi square $(3)=287.75, \mathrm{p}<.001)$, the two factor model $(\Delta$ chi-square $(5)=684.30, \mathrm{p}<$. $001)$ and the single factor model $(\Delta$ chi-square $(9)=1183.39, p<.001)$. The results show that the concept in this study has a good discriminant validity, and LML, as a multidimensional construct, has a good distinction with other concepts.

The descriptive statistical analysis results of this study include the mean value, standard deviation and Pearson correlation of each variable. LML was significantly correlated with feedback quality $(\mathrm{r}=.49, \mathrm{p}<.01)$, task performance $(\mathrm{r}=.68, \mathrm{p}<.01)$, peripheral performance $(\mathrm{r}=.34, \mathrm{p}<.01)$.

\subsection{Hypothesis testing}

In this study, boostrapping (samples=1000) method was used to estimate the total effect, indirect effect and direct effect of the above intermediary model. Confidence interval (CI) was set at $99 \%$. From LML to task performance and peripheral performance, there is a significant overall effect, namely, traded-task performance $=.46,99 \%$ CI:.28 .64, traded-peripheral performance $=.38,99 \%$ CI:.20 .56, which indicates that LML can significantly predict task performance and peripheral performance in a positive way. Hypothesis 1 and hypothesis 2 are all supported. Hypothesis 4 holds that feedback quality can mediate the relationship between LML and task performance, but the indirect effect estimated by boostrapping does not support this hypothesis. The indirect effect of feedback quality on this path is not significant, i.e. Hypothesis 5 holds that feedback quality can 
mediate the relationship between LML and surrounding performance. The indirect effect estimated by boostrapping supports this hypothesis. The path coefficient between LML and feedback quality is equal to.70, $\mathrm{p}<.001$, indicating that LML can significantly positively predict the feedback quality perceived by employees. Hypothesis 3 is supported.

\section{Research discussion and enlightenment}

In this study, a latent variable intermediary model was constructed, and the indirect effects of the model were estimated by using boostrapping method. The analysis results basically supported the theoretical framework and model of this study. In general, the study obtained results from three aspects:

First of all, the data supports the positive influence of LML on employee task performance and peripheral performance. When the leader shows motivational speech behavior, it can promote subordinates to improve task performance and peripheral performance. Secondly, within the framework of the organizational communication theory, the communication process of leaders to subordinates can trigger the subordinates' evaluation of their speech behaviors. The important way to measure the effectiveness and credibility of leaders' speech lies in the quality of their feedback to subordinates' demands, and even whether they can construct a good two-way communication and feedback environment. Finally, the most important result of this study is that the perceived feedback quality of employees as an intermediary variable can explain how LML affects the peripheral performance and task performance of subordinates.

The conclusion of this study provides favorable evidence to uncover the "black box" between the leadership speech behavior and the employee's work performance (especially the peripheral performance), and has certain enlightenment to the enterprise's human resource management practice. Organizational practice needs to pay attention to and train the way and content of leadership expression, improve the directionality, emotion and significance of leadership discourse, and improve the motivation of employees through leadership language management and training.

\section{References}

[1] Madlock, P. E. (2013). The influence of motivational language in the technologically mediated realm of telecommuters [J]. Human Resource Management Journal, 23(2):196-210.

[2] Sullivan, J. J. (1988). Three roles of language in motivation theory [J]. Academy of Management Review, 13(1) : 104-115.

[3] Eisenberg, E. M. (2009). Organizational communication theories [C]. In S. W. Littlejohn \& K. A. Foss (Eds.), Encyclopedia of communication theory (pp. 701-706). Thousand Oaks, CA: SAGE Publications, Inc.

[4] Mayfield, J., \& Mayfield, M. (2012). The relationship between leader motivating language and self-efficacy: A partial least squares model analysis [J]. Journal of Business Communication, 49(4): 357-376.

[5] Brower, H. H., Fiol, C. M., \& Emrich, C. G. (2007). The language of leaders [J]. Journal of Leadership Studies, 1(3): 67-80.

[6] Mayfield, J. R., Mayfield, M. R., \& Kopf, J. (1998). The effects of leader motivating language on subordinate performance and satisfaction [J]. Human Resource Management, 37(3-4): 235-248.

[7] Mayfield, J., \& Mayfield, M. (2007). The effects of leader communication on a worker's intent to stay: An investigation using structural equation modeling [J]. Human Performance, 20(2): 85102.

[8] Wang, C. W., Fan, K. T., Hsieh, C. T., \& Menefee, M. L. (2009). Impact of motivating language on team creative performance [J]. Journal of Computer Information Systems, 50(1): 133-140. 
[9] Mayfield, J., \& Mayfield, M. (2009). The role of leader motivating language in employee absenteeism [J]. Journal of Business Communication, 46(4): 455-479.

[10] Salancik, G. R., \& Pfeffer, J. (1978). A social information processing approach to job attitudes and task design [J]. Administrative Science Quarterly, 23(2): 224-253. 